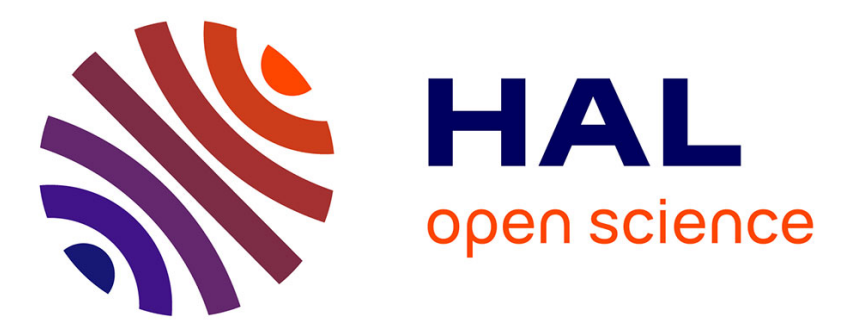

\title{
Improvement of the chemical content prediction of a model powder system by reducing multiple scattering using polarized light spectroscopy
}

R. Bendoula, Alexia Gobrecht, B. Moulin, J.M. Roger, Véronique Bellon

Maurel

\section{To cite this version:}

R. Bendoula, Alexia Gobrecht, B. Moulin, J.M. Roger, Véronique Bellon Maurel. Improvement of the chemical content prediction of a model powder system by reducing multiple scattering using polarized light spectroscopy. Applied Spectroscopy, 2015, 69 (1), pp.95-102. 10.1366/14-07539 . hal-01152872

\section{HAL Id: hal-01152872 \\ https://hal.science/hal-01152872}

Submitted on 18 May 2015

HAL is a multi-disciplinary open access archive for the deposit and dissemination of scientific research documents, whether they are published or not. The documents may come from teaching and research institutions in France or abroad, or from public or private research centers.
L'archive ouverte pluridisciplinaire HAL, est destinée au dépôt et à la diffusion de documents scientifiques de niveau recherche, publiés ou non, émanant des établissements d'enseignement et de recherche français ou étrangers, des laboratoires publics ou privés. 


\section{Improvement of the chemical content prediction of a model}

\section{powder system by reducing multiple scattering using polarized}

3

9 Abstract: Near Infrared Spectroscopy (NIRS) is a powerful non destructive

\section{light spectroscopy}

\author{
Ryad Bendoula, ${ }^{1, *}$ Alexia Gobrecht, ${ }^{1}$ Benoit Moulin, ${ }^{1}$ Jean-Michel Roger ${ }^{1}$ \\ and Veronique Bellon-Maurel ${ }^{1}$ \\ ${ }^{1}$ Irstea, UMR ITAP, 361 rue J-F Breton, F-34196 Montpellier, France \\ ryad.bendoula@irstea.fr
}

and requiring no sample preparation. It is widely used in routine analysis and also in-

line in industries, in-vivo with biomedical applications or in-field for agricultural and environmental applications. However, highly scattering samples subvert BeerLambert law's linear relationship between spectral absorbances and the concentrations. Instead of spectral pre-processing, which is commonly used by NIR spectroscopists to mitigate the scattering effect, we put forward an optical method, i.e. coupling polarized light with NIR spectrometry, to free spectra from scattering effect. This should allow us to retrieve linear and steady conditions for spectral analysis. When tested in Visible-NIR (VIS-NIR) range (400-800 $\mathrm{nm}$ ) on model media - mixtures of scattering and absorbing particles - the set-up provided 
significant improvements in absorber concentration estimation precision as well as in the quality and robustness of the calibration model.

Index Heading: Polarized Light Spectroscopy, Multiple Light Scattering, Diffuse

24 Reflectance, Optical Properties, Chemical Content Prediction.

\section{Introduction}

Visible - Near infrared spectroscopy (VIS-NIRS) is a well-known technique used for measuring the chemical composition of a wide variety of media and products. Although VIS-NIRS has been quoted in articles for approximately 50 years ${ }^{1,2}$ with this purpose, it really took off in the late 80 's in agricultural and food applications (jumping from around 10 publications per year in the late 80 's to 150 publications per year in the turn of century), and then in the 90's for pharmaceutical and biomedical applications. Today, it plays a major role in these sectors, as a routine laboratory method for in vivo or in-line monitoring system. On the one hand VIS-NIRS presents several advantages: VIS-NIR extinction coefficients are small compared to midinfrared (MIR) ones, which allows light to penetrate deeper into objects and avoids time-consuming sample preparation; VIS-NIR light scattering makes it possible to analyze bulk samples with a retro-diffusion optical configuration, thus turning it into a non-destructive technique. In addition VIS-NIR optical components are low cost and with high Signal-to-Noise Ratio (SNR). On the other hand, VIS-NIRS has several drawbacks: the VIS-NIR spectrum is poorly resolved as it is made up of scattering effects and of wide low-intensity harmonics and combinations of MIR 
42 fundamental absorption bands. Consequently, retrieving chemical information from

43 VIS-NIR spectra is quite painstaking and requires advanced chemometrics: it is

44 based on calibration models to be built between VIS-NIR spectra and known

45 concentrations of a set of calibration samples. Traditionally, linear multivariate

46 calibration methods such as Principal Component Regression (PCR) and Partial

47 Least Square Regression (PLSR) are used in VIS-NIRS. However, scattering effects

48 are troublesome in the VIS-NIR spectra of turbid media, defined by Shi and

49 Anderson $^{3}$ as "samples exhibiting multiple scattering events". Scattering can be

50 several orders of magnitude larger than absorption ${ }^{4}$ and may invalidate the use of

51 such data processing methods, which are themselves based on the underlying

52 assumption of a linear Beer-Lambert law relationship between absorbance spectra

53 and chemical concentration ${ }^{5}$. It is therefore necessary for VIS-NIR spectroscopists

54 working on highly scattering media to use strategies to release VIS-NIR spectra from

55 scattering effects. The most common strategy is spectral pre-treatment. These

56 preprocessing step is specifically designed to reduce multiplicative and additive

57 effects caused by variations in sample physical properties ${ }^{6,7}$. Among them, standard

58 normal variate (SNV) often associated to detrend ${ }^{8}$, multiplicative signal correction

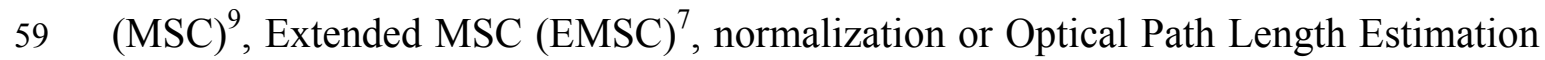

60 and Correction (OPLEC) $)^{5,10}$. However, these approaches remain questionable: they

61 consider that scattering is nearly constant over the wavelengths, which is not the

62 case $^{3}$; they may eliminate chemical-related information, which is very small with 
63 regard to scattering effects ${ }^{11}$; they are inappropriate when light scattering varies 64 greatly from sample to sample ${ }^{12}$.

65 Another option is to acquire the spectrum in a way that separates the part related to 66 absorption from the part related to scattering. Specific experimental techniques, 67 related to the application of light propagation theory and resolution of the Radiative 68 Transfer Equation ${ }^{3}$ have been proposed, including adding-doubling set-ups ${ }^{12-14}$, 69 spatially-resolved spectroscopy ${ }^{15}$, time-resolved spectroscopy ${ }^{4,16}$ and frequency70 resolved spectroscopy ${ }^{17}$.

71 Although powerful, these methods have their limitations, particularly when applied 72 on highly turbid samples. First, they may require complex and sometimes expensive 73 optical implementations, which may not be compatible with conventional complex media ${ }^{12,18}$.

Whereas separating absorption and scattering from a VIS-NIR signal is still an open research issue on highly turbid samples, the main demand from VIS-NIR spectroscopists is merely for spectra with reduced impact of scattering in order to

83 better fit Beer-Lambert's Law conditions ${ }^{19,20}$. 
84 Light polarization subtraction is a simple technique to reduce directly the effects of

85 multi-scattering on the measured signal ${ }^{20,21}$. This approach has been based on the fact

86 that, when light interacts with matter, a small number of scattering events do not

87 significantly modify the polarization status of the beam whereas multiple scattering

88 leads to depolarization ${ }^{18,22}$. Polarization subtraction technique ${ }^{19,23-25}$ was used to

89 select light beams that retain initial polarization and which are therefore less

90 impacted by multiple scattering events.

91 Although this technique has gained interest in the field of biomedical ${ }^{20,21,25}$, where it

92 is used to optically target subsurface organelles (particles suspended in water) and

93 tissues (layered samples), it is either poorly understood or not used by NIR

94 spectroscopists working with agricultural, food, pharmaceutical and other industrial

95 samples. To our knowledge, polarized NIRS techniques have never been applied to

96 routine or in-line analysis to reduce scattering effects on spectra on turbid media.

97 In this paper, the effectiveness of this multi-scattering correction based on the

98 polarization subtraction is evaluated using a two-component model powder system.

99 The objectives of this paper were to assess the effect of multi-scattering correction (i)

100 on the performances of a calibration model and (ii) on the robustness of the

101 prediction model built from the corrected spectra for predicting the absorber's

102 concentration of powder samples

\section{2. Theoretical Model : Polarization subtraction}


104 Capital bold characters will be used for matrices, e.g. X; non bold characters will be used for column vectors, e.g. X

106 Polarization subtraction technique ${ }^{23-25}$ is based on the polarization-maintaining property of weakly scattered light. When polarized light illuminates a scattering medium, weakly scattered light will emerge in its original polarization state ${ }^{21,26-28}$,

109 while multiple scattered light will emerge with random polarization. In the case of 110 linearly polarized source, the light that is remitted in the same polarization channel as 111 the input illumination is composed by light that has maintained its original 112 polarization state plus a component from the randomly polarized heavily scattered

113 light (cf equation 1). Light that emerges in the orthogonal polarization channel 114 contains only randomly polarized light, approximately equal to the randomly 115 polarized component in the original polarization state (cf equation 2).

$117 \quad I_{\perp}(\lambda)=\frac{\Omega}{2 \pi} \cdot I_{0}(\lambda) \cdot \beta(\lambda) \cdot M(\lambda)$

Where $I_{\|}(\lambda)$ and $I_{\perp}(\lambda)$ are the light scattered by the media with parallel and perpendicular polarization respect to the polarization of the illumination light. $\mathrm{I}_{0}(\lambda)$

122 is the intensity of the illumination light. $\Omega$ is the collection solid angle, residual 123 term of the integration on the solid angle ${ }^{23-25}$, of the optical device. $S(\lambda)$ and $M(\lambda)$ 124 are the probabilities of light undergoing single and multiple scattering respectively.

125 Since all light undergoes scattering : 
$S(\lambda)+M(\lambda)=1$

127

128

129

130

131

132

133

134

135

136

$\alpha(\lambda)+\beta(\lambda)=1$

34 equal to :

Finally, $\alpha(\lambda)$ and $\beta(\lambda)$ are the multiple light scattered ratio by the media with the parallel and perpendicular polarization respect to the polarization of the illumination light. The sum $\alpha(\lambda)$ and $\beta(\lambda)$ must be one:

By subtracting (1) to (2), the intensity of light undergoing single scattering $\left(\mathrm{I}_{\mathrm{ss}}(\lambda)\right)$ is

$I_{S S}(\lambda)=I_{\|}(\lambda)-I_{\perp}(\lambda)=\frac{\Omega}{2 \pi} \cdot I_{0}(\lambda) \cdot[S(\lambda)+(\alpha(\lambda)-\beta(\lambda)) \cdot M(\lambda)]$

In conclusion, the part of the single-scattering effect in the signal is preserved and the multi-scattering effect is highly reduced.

\section{Materials and Methods}

\subsection{Instrumentation}

In the experimental setup (figure 1), a halogen light source $(150 \mathrm{~W}$, Leica Cls ) was coupled with a $940 \mu \mathrm{m}$ core diameter optical fiber (numerical aperture (N.A) 0.22 , Sedi \& ATI). The light delivered by the fiber was collimated by an aspheric lens (F220SMA-B - Thorlabs). The incident beam was a $2 \mathrm{~cm}$ diameter circular spot with $1^{\circ}$ divergence. The incident and reflected beam were polarized thought two broadband (400 nm - 800nm) polarizers (NT52-557, Edmunds Optics). Incident light was 
148 linearly polarized and reflected light was collected in a narrow cone $\left(1^{\circ}\right)$. The output 149 from the analyzer was coupled inside an optical fiber (N.A 0.22 , Sedi \& ATI) by an 150 aspheric lens (F220SMA-B - Thorlabs). This fiber was connected to a spectrometer 151 (MMS1, Zeiss) featuring a detection range of $400 \mathrm{~nm}-800 \mathrm{~nm}$, with $3 \mathrm{~nm}$ resolution. A constant angle of $70^{\circ}$ was maintained between the excitation and collection arms. This angle was chosen to optimize intensity of the reflected beam and to avoid specular reflection.

\subsection{Experimental design and sample preparation}

156 Powdered samples mixing sand (Fontainebleau sand VWR International) and coloring dyes (brilliant blue FCF-E133 and chlorophyllin E141, purchased from

158 Colorey, respectively named E133 and E141 in the text) were prepared. Two sand particle size classes were used: $\mathrm{S}_{1}$ with a diameter less than $250 \mu \mathrm{m}$ and $\mathrm{S}_{2}$ with a

160 diameter greater than $250 \mu \mathrm{m}$. Sand play the role of a scattering but non absorbing matrix. One or both of the coloring dyes have been added at different densities to the sand, playing the role of absorbing substance in the mixture. Note that absorbers in powdered form also have scattering properties. Particle sizes of the coloring powders were less than $50 \mu \mathrm{m}$, with E133 being about three times smaller than E141.

165 Overall, 42 samples were prepared for spectral acquisition composing a calibration 166 set and 12 samples were prepared, afterward and with the same procedure to create 167 an independent test set.

168 The range of sample's colorant densities (in g.L-1) is specified in figure 2. 
169 Each sample was directly prepared in an airtight plastic container of $100 \mathrm{~mL}$ by

170 adding the precisely weighted corresponding amount of colorant in $20 \mathrm{~g}$ of sand

171 using an analytical balance (Kern 770). The maximum dye volume added was not

172 higher than $4 \%$ of the total sand volume. Considering that the pore volume for sand

173 is about $40 \%$, and that the dye particles are between 5 to 10 times smaller than the

174 sand, one can make the assumption that the dye would fill the interstices between the

175 sand particles and therefore not increase the initial volume of sand. The density of

176 the colorant $d_{\text {dye }}$ in a sample was obtained from :

$177 \quad d_{d y e}=\frac{m_{\text {dye }} \cdot d_{\text {sand }}}{m_{\text {sand }}}$

178 With $\mathrm{m}_{\text {dye }}$ the added mass of dye, $\mathrm{d}_{\text {sand }}$ the density of sand (which differ for $\mathrm{S}_{1}$ and

$\mathrm{S}_{2}$ ) and $\mathrm{m}_{\text {sand }}$ the mass of sand (here $20 \mathrm{~g}$ ). The colorant density ranged from [0 - 18

$180 \quad$ g. $\left.\mathrm{L}^{-1}\right]$.

181 To ensure homogeneity of the mixture, the sample was agitated after preparation and 182 again just before it was carefully transferred in an adapted $5 \mathrm{~cm}$ of diameter cup to 183 get an even and horizontal surface.

\subsection{Spectral acquisition}

185 For each sample, light was measured with the polarized spectrometer with parallel and perpendicular respect to the polarization of the illumination light. Dark current

$187\left(\mathrm{I}_{\mathrm{b}}\right)$ was recorded from all measured spectra and subtracted.

188 A broadband dielectric mirror (BB3-E02, Thorlabs) was used as a reference $\left(\mathrm{I}_{0}\right)$ to 189 standardize spectra from non-uniformities of all components of the instrumentation 190 (light source, fibers, lens, polarizer and spectrometer). 
191 From these measurements and the equation (5), a raw reflectance $\left(R_{w}\right)$ and a corrected reflectance $\left(\mathrm{R}_{\mathrm{c}}\right)$, for each sample, were calculated :

$$
\begin{aligned}
& \mathrm{R}_{\mathrm{w}}(\lambda)=\frac{\left(I_{\|}(\lambda)-I_{b \|}(\lambda)\right)+\left(I_{\perp}(\lambda)-I_{b \perp}(\lambda)\right)}{\left(I_{0 \|}(\lambda)-I_{b \|}(\lambda)\right)} \\
& \mathrm{R}_{\mathrm{c}}(\lambda)=\frac{\left(I_{\|}(\lambda)-I_{b \|}(\lambda)\right)-\left(I_{\perp}(\lambda)-I_{b \perp}(\lambda)\right)}{\left(I_{0 \|}(\lambda)-I_{b \|}(\lambda)\right)}=\frac{I_{S S}(\lambda)-\left(I_{b \|}(\lambda)+I_{b \perp}(\lambda)\right)}{\left(I_{0 \|}(\lambda)-I_{b \|}(\lambda)\right)}
\end{aligned}
$$

With $I_{\|}(\lambda)$ and $I_{\perp}(\lambda)$ the intensities of light scatters by the media with parallel and perpendicular polarization respect to the polarization of the illumination light and $\mathrm{I}_{0 \|}(\lambda)$ and $\mathrm{I}_{0 \perp}(\lambda)$, the intensities of light reflected by the standard mirror with parallel polarization respect to the polarization of the illumination light (as the perpendicular component emerging from the mirror is zero). $I_{b \|}(\lambda)$ and $I_{b \perp}(\lambda)$ are the dark current intensities recorded for each measurement.

\subsection{Multivariate analysis}

All computations and multivariate data analysis were performed with Matlab software v. R2012b (The Mathworks Inc., Natick, MA,USA).

\subsection{Linear unmixing}

208 The first step of Classical Least Square (CLS) ${ }^{29}$ was used to extract the reflectance 209 pure spectra of the absorbers from the reflectance spectra of the mixtures. Also 210 called K-matrix, this linear unmixing assumes that a spectrum is a linear combination 211 of the pure component's spectra. The whole calibration set $\mathbf{R}$ (42 samples mixing the 
212 absorbers at different concentrations) and C, the matrix of sample components

213 concentrations, were used to compute the linear least square estimated $\widehat{\mathbf{K}}$-matrix of

214 the two pure active components (E133 and E141) composing K knowing that:

$215 \widehat{K}=R C^{T}\left(C C^{T}\right)^{-1}$

216

217 Both the raw reflectance spectra $\left(\mathbf{R}_{\mathbf{w}}\right)$ and the corrected reflectance spectra $\left(\mathbf{R}_{\mathbf{c}}\right)$ were 218 used to compute respectively $\widehat{\mathbf{K}_{\mathbf{w}}}$ and $\widehat{\mathbf{K}_{\mathbf{c}}}$ containing the demixed pure spectra of $219 \quad$ E133 and E141.

\subsection{Calibration}

222 Partial Least Square (PLS) ${ }^{30}$ algorithm was used to model the chemical composition 223 of the powder mixture using $\mathbf{R}_{\mathbf{w}}$ and $\mathbf{R}_{\mathbf{c}}$. A general PLS model was built using the 224 whole calibration set (42 samples) to predict the samples of the independent test set 225 (12 samples). Secondly, to assess the robustness of the prediction models regarding 226 sand particle size, a PLS model was built with the samples set $\mathrm{S}_{2}$ and tested on the 227 independent test set $\mathrm{S}_{1}$ (figure 2 ). The number of latent variables was determined by 228 comparing performances by leave-one-out cross-validation ${ }^{31}$. Performances $\left(\mathrm{R}^{2}\right.$, 229 Standard Error of prediction corrected from the biais (SEPc)) and number of latent 230 variables of the different prediction models built with uncorrected and corrected 231 signals were compared when applied on the test set.

\section{Results and discussion}




\subsection{Spectra analysis}

235

\section{Bulk colorant}

The raw spectra $R_{w}(\lambda)$ and the corrected spectra $R_{c}(\lambda)$ of the pure powder colorant E133 and E141 are represented in figure 3 (a) and (b).

First comment is that the polarization subtraction reduces the global reflectance intensity of the measured signal (by 10 times). It is an expected result as only a small part of the signal is selected: the single-scattered one. Despite this reflectance loss, the corrected spectrum is not noisy and contains information about the sample.

Between $400 \mathrm{~nm}$ and $700 \mathrm{~nm}$, the raw spectrum and the corrected spectrum have similar shapes. For example, the spectroscopic signature of the colorant E133 appears to be purple (as seen in powdered form), mixing a reflectance peak at 450 $\mathrm{nm}$ (Blue) and at $650 \mathrm{~nm}$ (Red). However, these peaks are more marked in the corrected spectra. For the raw spectrum, crushing peaks can be explained by a strong increase in reflectance after $750 \mathrm{~nm}$. This sharp increase in reflectance, in a spectral range where the colorant does not absorb, is due to the multi-scattering. However, this effect seems to be less important for E141 than for E133. In the corrected spectra, this effect is strongly reduced (figure $3(\mathrm{~b})$ ).

\section{Sand and dye mixtures}

Figures 3 (c) and (d) show respectively the raw spectra and the corrected spectra of sand $\mathrm{S}_{1}$ mixing coloring powder E133 at different densities. When mixed with the colorant, sand is responsible for high multi-scattering as it is not absorbing the light. 
256 This physical phenomenon comes on top of the chemical information contained in 257 the spectra and masks the spectral features of the absorber. The shape of the raw 258 spectra of coloring powder E133 (figure 3 (a)) and the shapes of the raw reflectance

259 of the sand-dye mixture (figure 3 (c)) are completely different. The multiplicative 260 effect due to scattering is not wavelength dependent and depends on the number of 261 scatterers in the sample. Therefore, the more dye, the more scatterers and, 262 consequently the higher the reflectance. The order of the raw spectra is consistent 263 with the dye's concentration, but for a physical reason.

264 By applying the correction, the spectral features of the colorant are enhanced as it can be seen on figure 3 (d). The signature of the corrected spectra is similar to the spectral signal of the colorant E133 in powdered form (figure 3 (b)). The reflectance peaks at $450 \mathrm{~nm}$ and at $650 \mathrm{~nm}$ clearly appear, but more important, because linked to 268 the dye's concentration, the wavelength regions where absorbance occurs (400- 430 $\mathrm{nm}$ and $500-650 \mathrm{~nm}$ ) are now visible. In these regions, the spectrum ordering is 270 consistent with the dye concentration, contrary to the other areas where low absorbance occurs and reveals more complex reflectance patterns.

272 Figures 3 (e) and (f) show respectively the raw spectra and the corrected spectra of 273 sand $\mathrm{S}_{1}$ mixing this time coloring powder E141 at different densities. By comparing 274 the raw reflectance intensities of the sand - E133 mixtures (figure 3 (c)) and sand 275 E141 mixtures (figure 3 (e)), containing the same ranges of dye's densities, it appears that the level of $\mathrm{R}_{\mathrm{w}}$ is two-times higher for E133 than for E141. As stated in section

277 'Experimental design and sample preparation', the particle size of E141 is, at least, 
278 three-times larger than E133. This difference in particle diameter has a direct impact 279 on the elastic scattering phenomenon occurring during light-matter interaction. First, 280 for the same density of dye, small particles scatter more than larger particles ${ }^{21}$. 281 Secondly, the scattering angle differs between small and large particles: the larger the 282 diameter, the smaller the scattering angle. Combining these two properties, the 283 overall reflectance intensity will be higher for smaller particles, which is the case of raw reflectance of the Sand-E133 mixture.

285 For the corrected spectra $\mathrm{R}_{\mathrm{c}}$, there is no significant difference in the intensity level between sand - E133 mixtures (figure 3 (d)) and sand - E141 mixtures (figure 3 (f)).

This is coherent with the fact that the method corrects the spectra from multi288 scattering, which is mainly due to the sand particles but also, and in a significant manner, to the powdered colorant.

\subsection{Extraction of the absorber's pure spectra}

292 Figure (4) presents the demixed pure spectra $\widehat{\boldsymbol{K}}$ extracted from the reflectance spectra $293\left(\mathbf{R}_{\mathbf{w}}\right.$ and $\left.\mathbf{R}_{\mathbf{c}}\right)$. The linear unmixing (figure $\left.4(\mathrm{a})\right)$ applied to raw spectra $\left(\mathbf{R}_{\mathbf{w}}\right)$ provided 294 estimated pure spectra $\left(\widehat{\mathrm{K}_{\mathrm{W}_{-} \mathrm{E} 133}}(\lambda)\right.$ and $\left.\widehat{\mathrm{K}_{\mathrm{W}_{-} \mathrm{E} 141}}(\lambda)\right)$ that a very different from the 295 raw spectra $\mathrm{R}_{\mathrm{w}_{-} \mathrm{E} 133}(\lambda)$ and $\mathrm{R}_{\mathrm{w}_{-} \mathrm{E} 141}(\lambda)$ measured directly on the powders. The shape 296 of these spectra are not matching and the correlation coefficients, between $\widehat{\mathrm{K}_{\mathrm{w}_{-} \mathrm{E} 133}}(\lambda)$ and $\mathrm{R}_{\mathrm{w}_{-} \mathrm{E} 133}(\lambda)$ and between $\widehat{\mathrm{K}_{\mathrm{w}_{-} \mathrm{E} 141}}(\lambda)$ and $\mathrm{R}_{\mathrm{w}_{-} \mathrm{E} 141}(\lambda)$, are respectively equal to 0.59 and 0.74 . 
299 Linear unmixing assumes that a spectrum is a linear combination of the pure 300 components spectra. From these results, the failure to recover the absorber's pure 301 spectrum from the raw reflectance spectra, support the fact that interactions in the mixture are responsible of non-linearities which are directly responsible of the non-

303 linearities in the relationship between the absorbance and the absorbing power of the 304 sample. It is well known ${ }^{20,32}$ that scattering and absorbance are not independent 305 phenomenon. Scatter increases the mean free path of photons, which increases the 306 chances of being absorbed.

307 On the contrary, the computation of the $\widehat{\mathbf{K}}_{\mathbf{c}}$-matrix is successful and matches the pure 308 colorant corrected reflectance spectrum. Estimated pure spectra $\left(\widehat{\mathrm{K}_{\mathrm{C} \_ \text {E133 }}}(\lambda)\right.$ and $\left.309 \widehat{K_{C_{-} E 141}}(\lambda)\right)$ are very close to $R_{c_{-} E 133}(\lambda)$ and $R_{c_{-} E 141}(\lambda)$ measured directly on the 310 powder and corrected. The correlation coefficients are respectively 0.98 and 0.93 . As 311 the method corrects the signal from a physical phenomenon (multi-scattering), one can affirm that the observed interactions in the raw spectra are of optical nature (and not chemical interactions). Regarding these results and this consideration, 314 polarization subtraction correction induces, by reducing the multi-scattering effect, a 315 better linear relationship between the light attenuation and the absorption the dyes. 316 The recovered spectra $\widehat{\mathrm{K}_{\mathrm{c}}}$ are corrected from the physical interactions occurring in 317 the mixture.

\subsection{Calibration model}


Author-produced version of the article published in Applied Spectroscopy, 2015, №69(1), p.95-102

The original publication is available at http://www.ingentaconnect.com

http://dx.doi.org/10.1366/14-07539

321 Table 1 shows the quality parameter of the prediction models of the test set absorber's densities comparing the raw and the corrected spectra.

323 First, with the raw spectra $\mathrm{R}_{\mathrm{w}}(\lambda)$, the quality of the E133 prediction model is distinctly poorer than the quality of the E141 prediction model. The number of latent variables is much higher for E133 as well as the $\mathrm{SEP}_{\mathrm{c}}$. On the contrary, the figures of merit for the E141 model are good. Again, E133 and E141 behave differently. As stated before, multiscattering effect is more important when E133 is present in the mixture (with or without E141) because of a smaller particle diameter. Hence, nonlinearities between absorbance and absorber's concentration are more important and the PLS model is limited in building a performant linear prediction model for E133. When the models are built with the corrected reflectance spectra $R_{c}(\lambda)$, the figure of merit of the absorber's density prediction models are good and of the same level for E133 and E141. In both cases, the number of latent variables is decreasing. The improvement is less important for E141 but significant for E133. And both models have a lower standard error of prediction compared to the $R_{w}(\lambda)$ prediction models method mainly reduced the effect of multiscattering due to sand particles, enhancing the part of the signal related to chemical absorbance. In $\mathrm{R}_{w}(\lambda)$, while this information is present, it is masked by the multi-scattering and the PLS needs more latent

340 variable to extract this chemically related information to build a model.

341 Here, the different behavior of the two dyes is not obvious anymore. This agrees with

342 the hypothesis that the correction method equalizes the mean free photon path 
343 between all the samples, regardless of the particle size and shape of the sample's

344 constituents. For the two absorbers, the number of latent variables, which is an

345 indicator of the complexity of the models, is still high (respectively 5 and 4 for E133

346 et E141 with the corrected spectra $R_{c}(\lambda)$ ) for samples mixing only two different

347 absorbers. Theoretically, two PLS components should be sufficient. This agrees with

348 the initial assumption that the polarization subtraction method highly reduces the 349 multi-scattering but does not remove it completely. In addition, these results are 350 consistent with the conclusions of section 'Extraction of the absorber's pure spectra', 351 stating that the correction method restores, in a significant manner, the linear 352 relationship between the spectra and the absorber's density in the powdered samples.

353 To conclude, as the PLS models using the corrected spectra show good prediction 354 capacities, it fulfills the assumption that even if the corrected signal intensity is 355 highly reduced, the remaining information is of better quality in terms of signal 356 sensitivity.

\section{Robustness assessment}

359 Table 2 presents the results of the calibration model built with samples of one 360 particle size $\left(\mathrm{S}_{2}>250 \mu \mathrm{m}\right)$ and tested on samples with another particle size $\left(\mathrm{S}_{1}<250\right.$ $361 \mu \mathrm{m})$.

362 First, the models built with $\mathrm{R}_{\mathrm{w}}(\lambda)$ show, as previously observed, better predictions 363 for E141 than for E133, but, in overall, lower quality than in table 1. This confirms a 364 different behavior of E133 and E141, but also that the sand particle size has an effect 
365 in the quality of the predictions. A change in the physical structure of the samples

366 usually leads to low prediction performances because of the scattering impact on the

367 signal.

368 When built with the corrected spectra, again, the prediction of E133 highly improves,

369 while the gain is less significant for E141, which is also consistent with the previous

370 conclusions. But overall, the predictions are good, confirming that the corrected

371 spectra, composed by the single scattered part of the total reflectance signal, becomes

372 less dependent to physical changes in the sample.

373 The polarization subtraction method selects by optical means only, part of

374 information related to the powdered absorbers concentration, while discarding the 375 unwanted effect of multi-scattering on the signal. The measured signal becomes less 376 dependent of the particle size changes of the samples and therefore improves both 377 quality and robustness of the prediction models.

\section{5. Conclusion}

380 This study demonstrates the effectiveness of the polarized light subtraction method, 381 applied to a two component model powder system, which improves the performance 382 of multivariate calibration models.

383 By selecting only the light which has conserved the initial polarization and therefore 384 being less impacted by scattering events, the collected photons all have the same 385 mean free pathlength. As a consequence, the linear relationship between the spectra 386 and the absorbers concentration is restored. 
387 Then it is possible, by using Linear Unmixing to recover the pure spectra of the dyes.

388 These recovered spectra are corrected from the physical interactions occurring in the 389 mixture.

390 When the corrected spectra are used to build the PLS models, all the general quality 391 parameters and the parsimony improve, especially for highly scattering media. 392 Although the overall signal intensity is reduced after optical correction, the 393 remaining information in the corrected signal is sufficient and of better quality to 394 build a good prediction model, thus meaning that the signal sensitivity increases. 395 Inevitably there is a trade-off between making more accurate measurements and a 396 reduction of SNR.

397 After the polarization correction, the measured signal becomes less dependent to 398 physical changes (particle sizes) which also improve the robustness of the prediction 399 models.

400 This "plug and play" optical method offers the potential to be easy to implement to a 401 commercial spectrophotometry system and does not significantly increase the 402 measurement time.

\section{$404 \quad$ References and links}

405 (1) Hart, J.; Norris, K.; Golumbic, C. Cereal Chem 1962, 39, 94-99.

406 (2) Massie, D. R.; Norris, K. H. Transactions of the ASABE 1965, 8 (4), 598-600.

407 (3) Shi, Z.; Anderson, C. A. Journal of Pharmaceutical Sciences 2010, 99, 47664084783. 
(4) Chauchard, F.; Roger, J.; Bellon-Maurel, V.; Abrahamsson, C.; Andersson-

410 Engels, S.; Svanberg, S. Applied Spectroscopy 2005, 59, 1229-1235.

411 (5) Jin, J.-W.; Chen, Z.-P.; Li, L.-M.; Steponavicius, R.; Thennadil, S. N.; Yang, J.;

412 Yu, R.-Q. Analytical Chemistry 2012, 84, 320-326.

413 (6) Rinnan, A.; Berg, F. v. d.; Engelsen, S. B. TrAC Trends in Analytical Chemistry $4142009,28,1201-1222$.

415 (7) Martens, H.; Stark, E. Journal of Pharmaceutical and Biomedical Analysis 1991, $4169,625-635$.

417 (8) Barnes, R. J.; Dhanoa, M. S.; Lister, S. J. Appl. Spectrosc. 1989, 43, 772-777.

418 (9) Geladi, P.; MacDougall, D.; Martens, H. Appl. Spectrosc. 1985, 39, 491-500.

419 (10) Chen, Z.-P.; Morris, J.; Martin, E. Analytical Chemistry 2006, 78, 7674-7681.

420 (11) Martens, H.; Nielsen, J. P.; Engelsen, S. B. Analytical Chemistry 2003, 75, 394 421404.

422 (12) Steponavicius, R.; Thennadil, S. N. Analytical Chemistry 2011, 83, 1931-1937.

423 (13) Prahl, S. Optical-thermal response of laser-irradiated tissue, A 1995, 122-127.

424 (14) Steponavicius, R.; Thennadil, S. N. Analytical Chemistry 2009, 81, 7713-7723.

425 (15) Farrell, T.; Patterson, M.; Wilson, B. Medical Physics 1992, 19, 879-888.

426 (16) Abrahamsson, C.; Johansson, J.; Andersson-Engels, S.; Svanberg, S.; Folestad, 427 S. Analytical Chemistry 2005, 77, 1055-1059.

428 (17) Torrance, S.; Sun, Z.; Sevick-Muraca, E. Journal of Pharmaceutical Sciences 429 2004, 93,1879-1889. 
(18) Swartling, J.; Dam, J.; Andersson-Engels, S. Applied Optics 2003, 42, 4612-

431

432

433

434

435

436

437

438

439

440

441

442

443

444

445

446

447

448

449

450

451

4620.

(19) Hebden, J. C.; Arridge, S. R.; Delpy, D. T. Physics in Medicine and Biology 1997, 42, 825 .

(20) Lu, B.; Morgan, S. P.; Crowe, J. A.; Stockford, I. M. Appl. Spectrosc. 2006, 60, 1157-1166.

(21) Backman, V.; Gurjar, R.; Badizadegan, K.; Itzkan, I.; Dasari, R. R.; Perelman, L. T.; Feld, M. Selected Topics in Quantum Electronics, IEEE Journal of 1999, 5, 1019-1026.

(22) Abrahamsson, C.; Löwgren, A.; Strömdahl, B.; Svensson, T.; AnderssonEngels, S.; Johansson, J.; Folestad, S. Appl. Spectrosc. 2005, 59, 1381-1387.

(23) Schmitt, J.; Gandjbakhche, A.; Bonner, R. Appl. Opt. 1992, 31, 6535-6546.

(24) Morgan, S.; Ridgway, M. Optics Express 2000, 7, 395-402.

(25) Demos, S. G.; Alfano, R. R. Appl. Opt. 1997, 36, 150-155.

(26) Demos, S. G.; Alfano, R. R. Opt. Lett. 1996, 21, 161-163.

(27) Sokolov, K.; Drezek, R.; Gossage, K.; Richards-Kortum, R. Opt. Express $1999,5,302-317$.

(28) Yoo, K.; Alfano, R. Physics Letters A 1989, 142, 531-536.

(29) Geladi, P. Spectrochimica Acta Part B: Atomic Spectroscopy 2003, 58, 767 782.

(30) Wold, S.; Sjöström, M.; Eriksson, L. Chemometrics and Intelligent Laboratory Systems 2001, 58, 109-130. 
452 (31) Wold, S. Technometrics 1978, 20, 397-405.

453 (32) Stockford, I. M.; Lu, B.; Crowe, J. A.; Morgan, S. P.; Morris, D. E. Appl.

454 Spectroscopy 2007, 61, 1379-1389. 


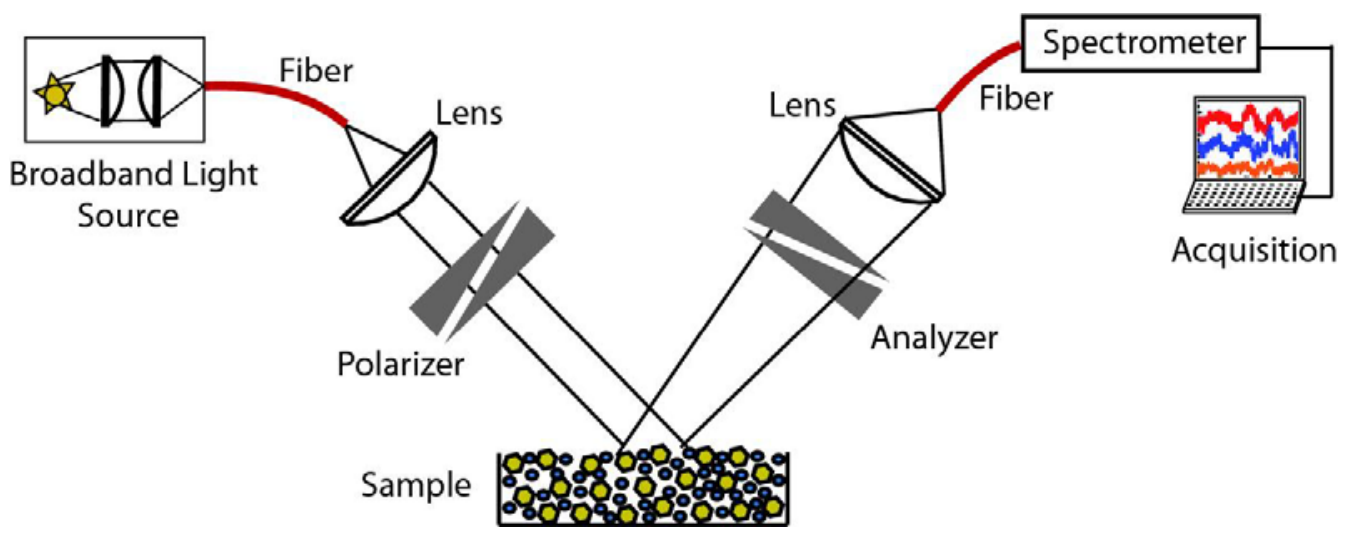

Figure 1: Schematic diagram of polarized light spectroscopy system.

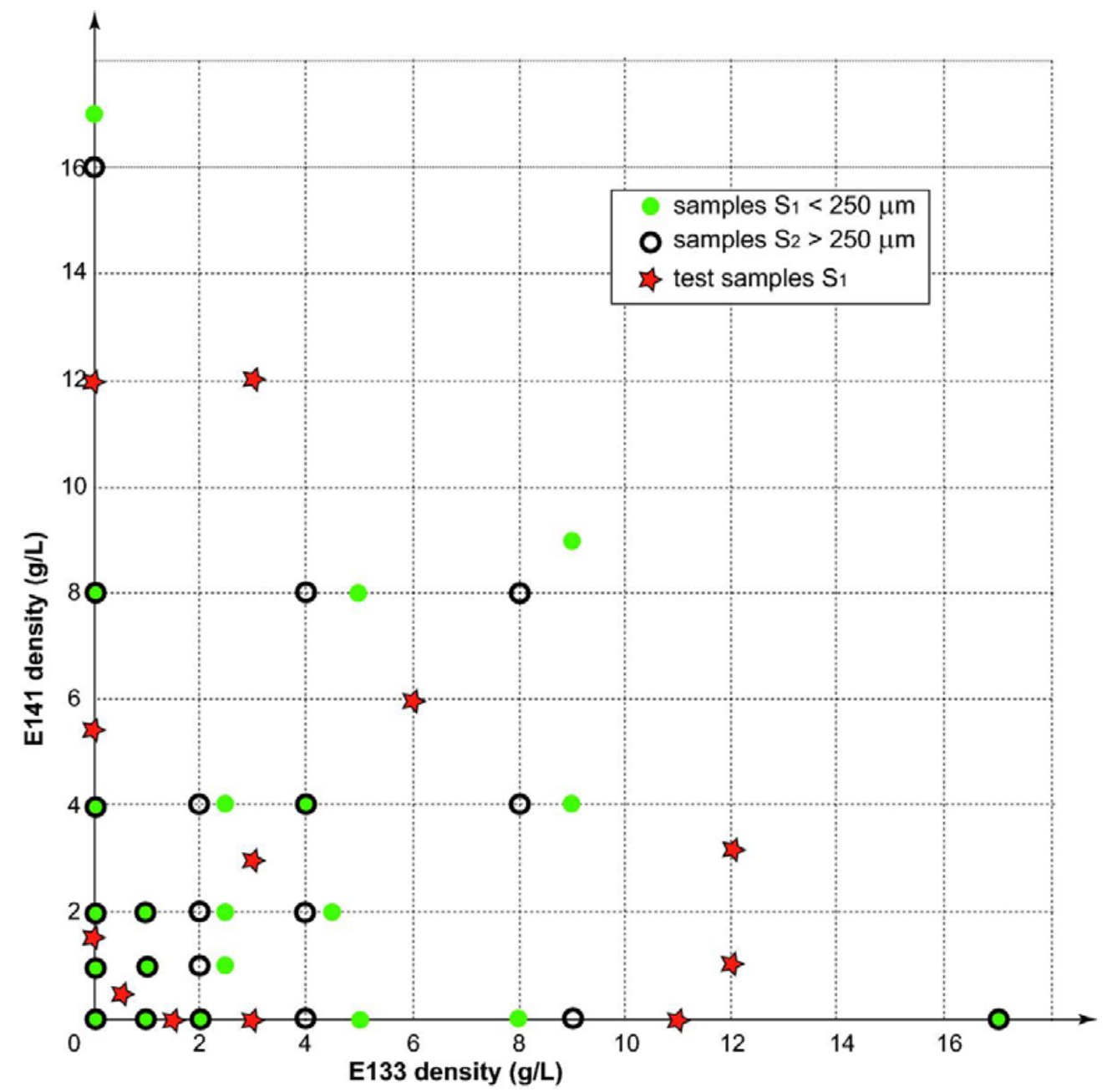

Figure 2: Experimental design presenting the dye densities in g.L $\mathrm{L}^{-1}$ of 42 samples for the calibration set and 12 samples for the independent test set. 

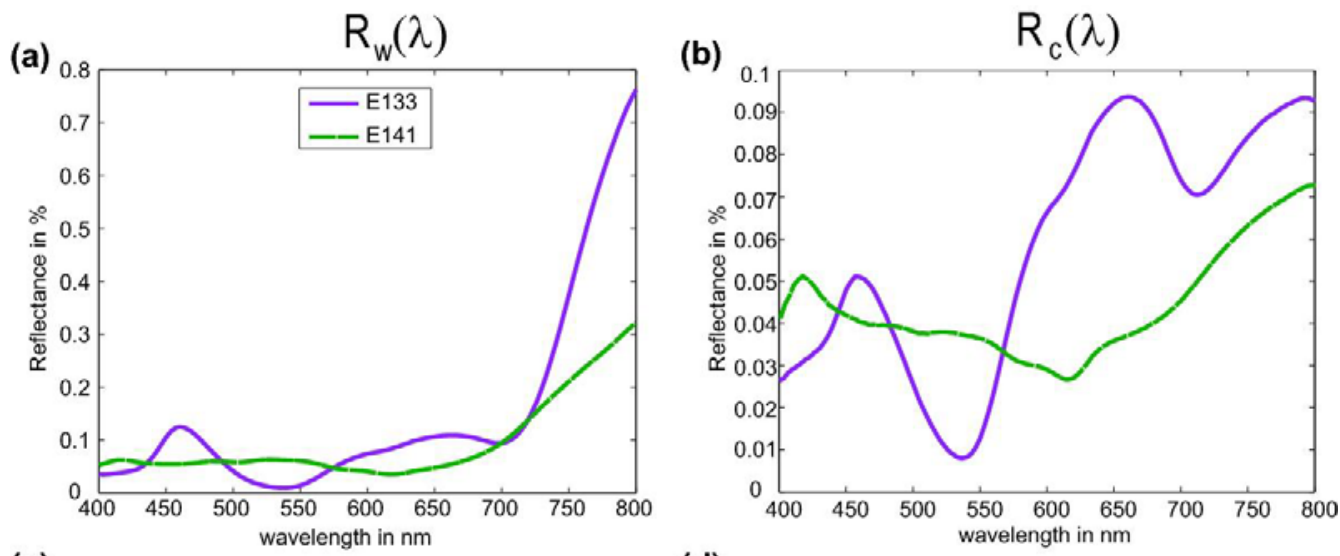

(c)



(d)



(e)

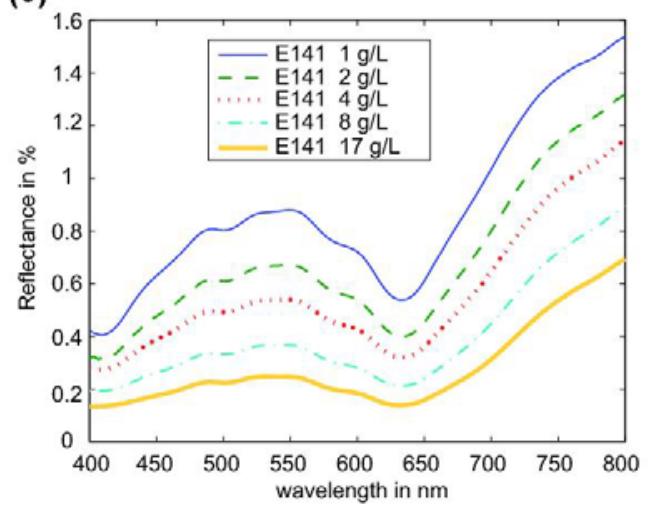

(f)



Figure 3: (a) Raw reflectance spectra of coloring powder E133 and E141. (b) Corrected reflectance spectra of coloring powder E133 and E141 (c) Raw reflectance spectra of sand $\mathrm{S}_{1}+$ coloring powder E133 mixed at different densities. (d) Corrected reflectance spectra of sand $\mathrm{S}_{1}+$ coloring powders E133 mixed at different densities.(e) Raw reflectance spectra of sand $\mathrm{S}_{1}+$ coloring powder E141 mixed at different densities.(f) Corrected reflectance spectra of sand $\mathrm{S}_{1}+$ coloring powders E141 mixed at different densities. 


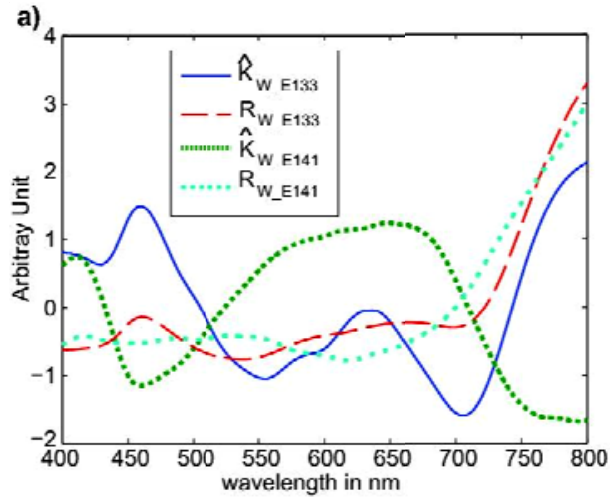

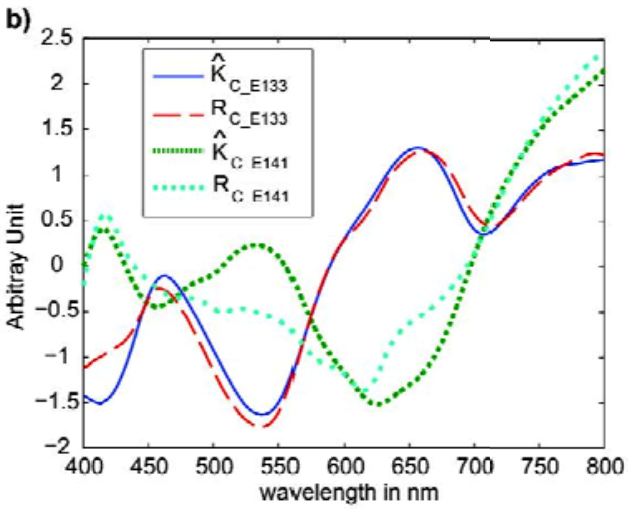

Figure 4 : Comparison of the raw and the corrected spectra acquired on the two coloring powders $\left(\mathrm{R}_{\mathrm{w}_{-} E 133}(\lambda), \mathrm{R}_{\mathrm{w}_{-} E 141}(\lambda)\right.$ and $\left.\mathrm{R}_{\mathrm{c}_{-} \mathrm{E} 133}(\lambda), \mathrm{R}_{\mathrm{c}_{-} \text {E141 }}(\lambda)\right)$ with the demixed pure spectrum $\left(\mathrm{K}_{\mathrm{w}_{-} \mathrm{E} 133}(\lambda), \mathrm{K}_{\mathrm{W} \_ \text {E141 }}(\lambda)\right.$ and $\left.\mathrm{K}_{\mathrm{c}_{-} \text {E133 }}(\lambda), \mathrm{K}_{\mathrm{c}_{-} \text {E141 }}(\lambda)\right)$ extracted respectively from $\left(\mathbf{R}_{\mathbf{w}}\right)$ and $\left(\mathbf{R}_{\mathbf{c}}\right)$ with Linear unmixing.

Table 1: Figure of merit of the calibration models

\begin{tabular}{|c|c|c|c|c|c|c|c|}
\hline & \begin{tabular}{|l} 
Cal set \\
(n)
\end{tabular} & $\begin{array}{l}\text { Test set } \\
\text { (n) }\end{array}$ & Spectra & $\begin{array}{l}\text { Predicted } \\
\text { absorber }\end{array}$ & $\begin{array}{l}\text { PLS } \\
\text { factors }\end{array}$ & $\mathrm{R}^{2}$ & $\begin{array}{l}\text { SEPc } \\
\left(\% \text { g.L }{ }^{-1}\right)\end{array}$ \\
\hline \multirow{2}{*}{$\begin{array}{l}\text { General } \\
\text { model }\end{array}$} & \multirow{2}{*}{$\begin{array}{c}\mathrm{S}_{1}+\mathrm{S}_{2} \\
(42)\end{array}$} & \multirow{2}{*}{$\begin{array}{l}\mathrm{S}_{1} \\
\text { (12) }\end{array}$} & $\mathbf{R}_{\mathbf{w}}$ & $\begin{array}{l}\text { E133 } \\
\text { E141 }\end{array}$ & $\begin{array}{l}8 \\
5 \\
\end{array}$ & $\begin{array}{l}0.75 \\
0.91 \\
\end{array}$ & $\begin{array}{l}2.52 \\
1.3\end{array}$ \\
\hline & & & $\mathbf{R}_{\mathrm{c}}$ & $\begin{array}{l}\text { E133 } \\
\text { E141 }\end{array}$ & $\begin{array}{l}5 \\
4\end{array}$ & $\begin{array}{l}0.91 \\
0.93\end{array}$ & $\begin{array}{l}1.41 \\
1.12\end{array}$ \\
\hline
\end{tabular}

Table 2: Figure of merit of the calibration model built with samples of one particle size $\left(\mathrm{S}_{2}\right)$ and tested on samples with another particle size $\left(\mathrm{S}_{1}\right)$

\begin{tabular}{|c|c|c|c|c|c|c|c|}
\hline & $\begin{array}{l}\text { Cal set } \\
\text { (n) }\end{array}$ & \begin{tabular}{|l} 
Test set \\
(n)
\end{tabular} & Spectra & $\begin{array}{l}\text { Predicted } \\
\text { absorber }\end{array}$ & $\begin{array}{l}\text { PLS } \\
\text { factors }\end{array}$ & $\mathrm{R}^{2}$ & $\begin{array}{l}\text { SEPc } \\
\left(\% \text { g.L } L^{-1}\right)\end{array}$ \\
\hline \multirow{2}{*}{$\begin{array}{l}\text { General } \\
\text { model }\end{array}$} & \multirow{2}{*}{$\begin{array}{l}\mathrm{S}_{2} \\
\text { (21) }\end{array}$} & \multirow{2}{*}{$\begin{array}{l}\mathrm{S}_{1} \\
(12)\end{array}$} & $\mathbf{R}_{\mathrm{w}}$ & $\begin{array}{l}\text { E133 } \\
\text { E141 }\end{array}$ & $\begin{array}{l}5 \\
5\end{array}$ & $\begin{array}{l}0.69 \\
0.83\end{array}$ & $\begin{array}{l}3.33 \\
1.79\end{array}$ \\
\hline & & & $\mathbf{R}_{\mathrm{c}}$ & $\begin{array}{l}\text { E133 } \\
\text { E141 }\end{array}$ & $\begin{array}{l}4 \\
3\end{array}$ & $\begin{array}{l}0.91 \\
0.93\end{array}$ & $\begin{array}{l}1.72 \\
1.73\end{array}$ \\
\hline
\end{tabular}

